\title{
Longitudinal immunological characterization of the first presensitized recipient of a face transplant
}

\author{
Thet Su Win, ${ }^{1,2}$ Naoka Murakami, ${ }^{3}$ Thiago J. Borges, ${ }^{3}$ Anil Chandraker, ${ }^{3}$ George Murphy, ${ }^{4}$ \\ Christine Lian, ${ }^{4}$ Victor Barrera, ${ }^{5}$ Shannan Ho Sui, ${ }^{5}$ David Schoenfeld, ${ }^{6}$ Jessica Teague, ${ }^{2}$ \\ Ericka Bueno,, Stefan C. Tullius,, ${ }^{7}$ Bohdan Pomahac, ${ }^{1}$ Rachael A. Clark,, ${ }^{2}$ and Leonardo V. Riella ${ }^{3}$ \\ 'Division of Plastic Surgery, Department of Surgery, ${ }^{2}$ Department of Dermatology, ${ }^{3}$ Schuster Transplantation Research \\ Center, Renal Division, and ${ }^{4}$ Department of Pathology, Brigham and Women's Hospital, Harvard Medical School, Boston, \\ Massachusetts, USA. ${ }^{5}$ Bioinformatics Core, Department of Biostatistics, Harvard T.H. Chan School of Public Health, \\ Boston, Massachusetts, USA. ${ }^{6}$ Department of Biostatistics, Massachusetts General Hospital, Harvard Medical School, \\ Boston, Massachusetts, USA. 'Division of Transplant Surgery, Department of Surgery, Brigham and Women's Hospital, \\ Harvard Medical School, Boston, Massachusetts, USA.
}

Rejection affects greater than $\mathbf{8 0 \%}$ of face transplants, yet no diagnostic criteria for antibodymediated rejection (AMR) following face transplantation have been established. Given that different treatment strategies are required to address AMR and T cell-mediated rejection (TCMR), there is a critical need to delineate the features that can differentiate these two alloimmune responses. Here, we report the longitudinal immunological examination of what we believe to be the first and only highly sensitized recipient of a crossmatch-positive face transplant up to 4 years following transplantation. We conducted gene expression profiling on allograft biopsies collected during suspected AMR and TCMR episodes as well as during 5 nonrejection time points. Our data suggest that there are distinctive molecular features in AMR, characterized by overexpression of endothelial-associated genes, including ICAM1, VCAM1, and SELE. Although our findings are limited to a single patient, these findings highlight the potential importance of developing and implementing molecular markers to differentiate AMR from TCMR to guide clinical management. Furthermore, our case illustrates that molecular assessment of allograft biopsies offers the potential for new insights into the mechanisms underlying rejection. Finally, our medium-term outcomes demonstrate that face transplantation in a highly sensitized patient with a positive preoperative crossmatch is feasible and manageable.

Authorship note: T.S. Win and N. Murakami are co-first authors.

Conflict of interest: The authors have declared that no conflict of interest exists.

Submitted: March 16, 2017

Accepted: May 19, 2017

Published: July 6, 2017

Reference information: JCI Insight. 2017;2(13):e93894. https://doi.org/10.1172/jci. insight. 93894.

\section{Introduction}

An estimated 7 million people each year in the USA could benefit from vascularized composite allotransplants (VCAs) because of traumatic injuries, congenital anomalies, or surgical resection of tumors (1). Because VCA provides equal composite substitutes to the missing or deformed parts, it offers unprecedented functional and aesthetic restoration when compared with conventional reconstruction (2-4). At present, approximately 200 VCAs have been performed worldwide, including face, hand, abdominal wall, lower extremity, trachea, and genitourinary transplants (5).

Immune rejection is the major barrier preventing wider implementation of VCA transplants. Despite potent systemic immunosuppression, approximately $85 \%$ of all patients experience at least one episode of acute skin rejection in the first year after transplantation, and as many as $56 \%$ experience multiple episodes (6). Currently, diagnosis of rejection in skin-containing VCAs is confirmed by histological analyses of allograft skin biopsies according to the 2007 Banff classification (7), which is based on the location and intensity of inflammatory infiltrates. Thus, the current universally used classification of VCA rejection is based exclusively upon the histological features of cellular rejection, and to date, diagnostic criteria for antibody-mediated rejection (AMR) in VCA transplants have not been established.

In solid organ transplantation, diagnosis of AMR relies on 4 criteria: clinical evidence of acute graft dysfunction, histological features, immunopathological evidence of the action of antibodies (such as 
Table 1. Donor and recipient characteristics

\begin{tabular}{lcc}
\hline & Recipient & Donor \\
Age at transplant (yr) & 45 & 56 \\
Sex & Female & Female \\
Ethnicity & European descent & European descent \\
Mechanism of injury & Chemical burn & \\
Facial allograft details & Full face & \\
Panel reactive antibodies (\%) & 98 & \\
Donor-specific antibodies & Positive & \\
HLA mismatch (A, B, C, DR, DQ, DP) & 11 & \\
CMV status & Positive & Negative \\
EBV status & Positive & Positive \\
Ischemia time & & 3 hours
\end{tabular}

complement $4 \mathrm{~d}$ [C4d] deposition in the allograft), and serological evidence of donor-specific antibody (DSA) (8). However, a significant proportion of AMR cases are C4d negative (9-11), and biopsies with histological features of AMR or positive C4d staining do not always have detectable serum DSAs (12). Consequently, the exact combination of criteria required to diagnose AMR differs depending on the allograft type. For example, the 2013 International Society for Heart and Lung Transplantation Working Formulation restricts the diagnosis of AMR in heart transplants to histological and immunopathological criteria, without the requirement for serologic or clinical components (13). In renal transplantation, the 2013 Banff diagnosis of AMR requires histological evidence of tissue injury, the presence of circulating DSA, and evidence of current/recent antibody interaction with vascular endo-

thelium as well as, notably, removed C4d deposition as a requirement for diagnosis of AMR (14). Importantly, the 2013 Banff classification heralded a new era in rejection diagnostics by including molecular diagnostics as a diagnostic criterion for AMR in renal transplants (14).

Whether molecular assessments can be similarly established as diagnostic criterion for AMR following VCA transplants is unknown. In VCA transplants, development of DSAs following transplantation has been reported, but not all circulating DSAs are associated with $\operatorname{AMR}(15,16)$. In addition, the correlation between C4d deposition in VCA allograft skin and the presence of DSAs is unclear (17); positive C4d staining has been reported in the absence of DSAs $(18,19)$, and conversely, no C4d staining was detected in the skin of one VCA recipient despite the presence of circulating DSAs (20). Additionally, C4d deposition has been reported in inflammatory dermatoses unrelated to rejection (21). Establishment of consensus regarding diagnostic criteria is critically needed to determine the prevalence and effect of AMR on VCA transplants as well as for improved understanding of mechanisms underlying antibody-mediated graft injury and its optimal treatment strategy.

We previously reported the perioperative clinical management of a highly presensitized recipient who received a preoperative crossmatch-positive full-face transplant (22). Here, we report the immunological outcome of this patient for the first 4 years following transplantation. Following an episode of suspected AMR in the early period after transplant, the patient subsequently developed 3 episodes of $\mathrm{T}$ cell-mediated rejection (TCMR). We outline here the different treatment strategies used to resolve each of the rejection episodes. The availability of facial allograft biopsies and peripheral blood samples from the patient during rejection episodes and nonrejection time points allowed us to perform a unique, in-depth longitudinal immunological examination at both the cellular and molecular levels. We conducted gene expression profiling on the allograft biopsies collected during the suspected AMR and TCMR episodes as well as during 5 nonrejection time points. Our data suggest that there are distinctive features of AMR compared with TCMR in facial allograft, raising the possibility that molecular diagnostics may complement the clinical, serological, and histological examination in diagnosing AMR in face transplants.

\section{Results}

A 45-year-old highly sensitized female (Table 1) who sustained burns to $80 \%$ of total body surface area was transplanted with a full-face allograft from a $\mathrm{T}$ and B cell crossmatch-positive donor (22). Despite plasmapheresis, alongside the standard induction regimen with antithymocyte globulin antibodies, clinical evidence of rejection was noted on postoperative day 5 , with a corresponding infiltration of mononuclear cells in the allograft (Figure 1), an increase in circulating DSAs (Figure 2), and strong C4d deposition within the allograft (22). AMR was suspected, and the patient was treated with a combined therapy regimen, including plasmapheresis, eculizumab, alemtuzumab, bortezomib, and steroids (Figure 3) (22). By 6 months after transplantation, the serum DSAs became undetectable, clinical appearance improved, and the histological rejection resolved (Figure 2 and Figure 3). 
A
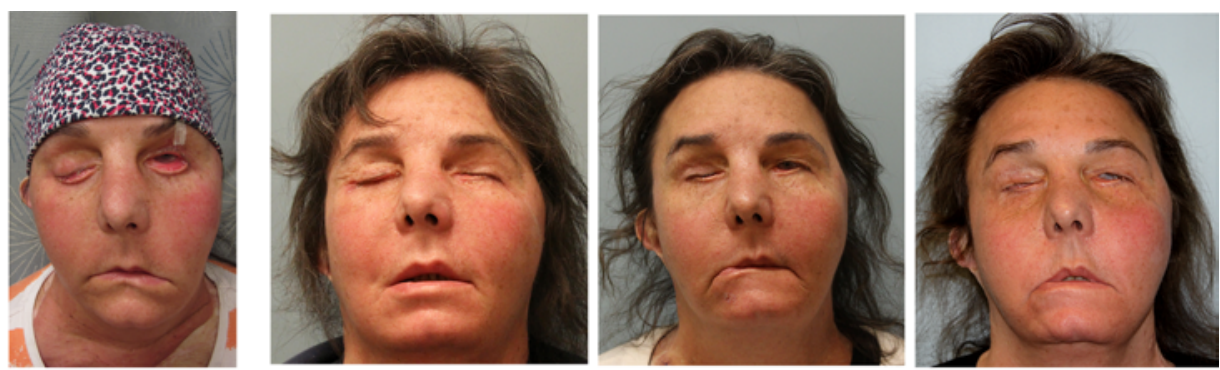

B

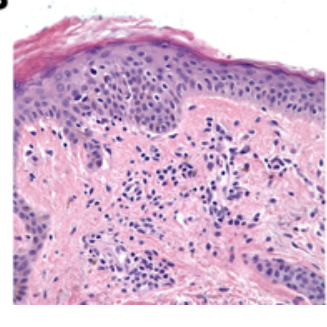

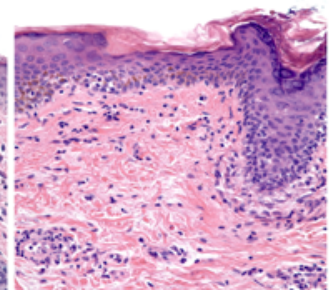
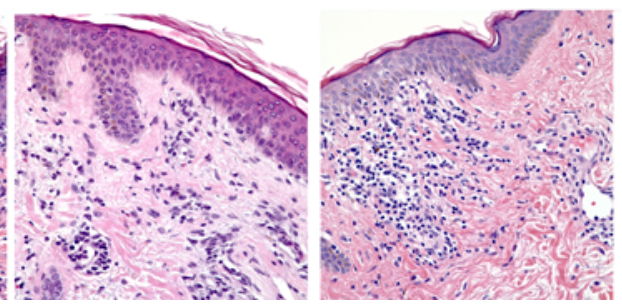

Figure 1. Clinical photographs and corresponding H\&E stainings of facial allograft skin biopsies during suspected AMR and TCMR episodes. (A) Clinically, the patient presented with erythema in the facial allograft, and (B) histological analysis showed dermal perivascular inflammatory infiltrates with foci of lymphocyte apposition to vacuolated basal keratinocytes and rare apoptotic keratinocytes in all 4 rejection episodes, regardless of the type of rejection. Original magnification, $\times 20$.

Following this suspected AMR episode, the patient experienced 3 episodes of TCMRs (Figure 1). At 12 months following transplantation, the patient presented with diffuse erythema, which was graded as Banff grade 3 rejection on histological examination (Figure 1, TCMR 1). No DSA was detected (Figure 2), and there was minimal C4d deposition (data not shown). Rejection resolved with $500 \mathrm{mg}$ solumedrol daily for 3 days, together with topical tacrolimus and clobetasol (Figure 3). At 21 months after transplantation, the allograft was noted to have diffuse erythema and edema, and histological examination of the allograft skin biopsy showed Banff grade 3 rejection (Figure 1, TCMR 2). No DSA was detected (Figure 2), and there was no C4d deposition within the allograft (data not shown). Rejection resolved with $100 \mathrm{mg}$ solumedrol daily for 5 days (Figure 3). At 24 months following transplantation, the patient presented with similar clinical signs, including diffuse erythema of the allograft (Figure 1, TCMR 3). DSA was negative (Figure 2), and there was no C4d deposition within the allograft skin (data not shown). Histological analysis showed Banff grade 2-3 rejection. The tacrolimus trough level was $8.2 \mathrm{ng} / \mathrm{ml}$. The rejection completely resolved with an increase in tacrolimus dose and topical application of clobetasol (Figure 3). As of the publication of this article, it had been 4 years since the patient underwent transplantation; the patient is currently managed with $1.5 \mathrm{mg}$ tacrolimus twice daily, $500 \mathrm{mg}$ mycophenolate mofetil twice daily, and $10 \mathrm{mg}$ prednisone daily and showing no signs of rejection (Supplemental Figure 2; supplemental material available online with this article; https://doi.org/10.1172/ jci.insight.93894DS1). Among infectious complications, the patient developed shingles, transient CMV viremia (220 IU/ml on PCR), and leg cellulitis. No other opportunistic infections have been observed in the after transplant period to date.

$A M R$ is associated with $B$ and $T$ follicular helper cell expansion in peripheral circulation. Longitudinal flow cytometric analysis of peripheral blood before and after transplantation was performed to capture the immunological signatures correlated with AMR or TCMR events (Figure 4). Consistent with our previous report (23), we observed an increased $\mathrm{T}$ effector population in the $\mathrm{CD} 8^{+}$population during $\mathrm{TCMR}$ episodes, but the regulatory $\mathrm{T}$ cell population was preserved (Supplemental Figure 1). Interestingly, both circulating $\mathrm{T}$ follicular helper (Tfh) cell $\left(\mathrm{CD} 4^{+} \mathrm{PD} 1^{+} \mathrm{CXCR} 5^{+}\right)$and memory B cell $\left(\mathrm{CD} 19^{+} \mathrm{CD} 27^{+}\right)$ populations increased 1 week after transplantation, concurrent with the suspected AMR episode but not during TCMR episodes (Figure 4). Notably, the proportion of circulating Tfh cells was markedly higher 1 week following transplantation, despite $\mathrm{T}$ cell depletion with antithymocyte globulin as induction therapy. The expansion of memory B cells, together with the increase in DSAs (Figure 2), suggests a dominant antibody-mediated response during the suspected AMR episode. 


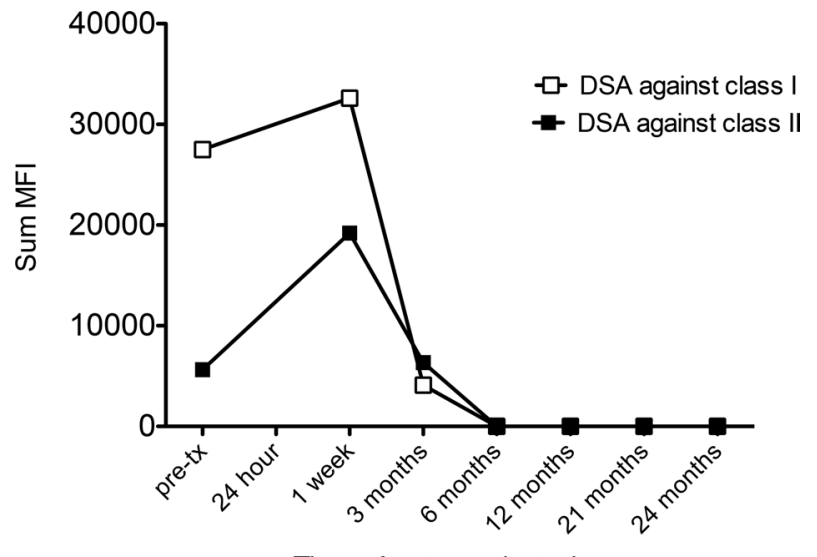

Time after transplantation
Figure 2. Sum mean fluorescence intensity of circulating donor-specific antibodies before and after facial transplantation. The sum mean fluorescence intensity (MFI) of circulating donor-specific antibodies (DSA) increased during the suspected AMR compared with that prior to transplantation. Following aggressive treatment, DSAs became undetectable and remained negative during the 3 subsequent episodes of TCMR. Pre-tx, before transplant.

Gene expression analysis of rejection biopsies versus nonrejection biopsies. We compared the gene expression profiles of rejection allograft biopsies (regardless of the type of rejection) with nonrejection biopsies. The timeline of collection of biopsies used for gene expression profiling is shown in Figure 5. Genes with an adjusted $P$ value of $\leq 0.1$ and a $\log _{2}$ fold change of $\geq 1$ were regarded as significant. Seventy-nine genes were upregulated and one gene was downregulated in the rejection biopsies compared with nonrejection biopsies (Supplemental Table 1). We observed activation of the IFN- $\gamma$ signaling pathway (including IRF1 and STAT1), genes responsible for recruitment of cytotoxic cells through production of chemokine ligands (primarily through the CXCR3/CCR5 pathway, including $C X C L 9$ and $C C L 5$ ), and genes with immune effector function (i.e., genes expressed by $\mathrm{CD} 8^{+}$cytotoxic $\mathrm{T}$ cells and natural killer cells upon activation, including GZMB) (Supplemental Table 1).

Gene expression analysis of biopsies collected during suspected AMR episodes versus TCMR episodes. Unsupervised principal component analysis performed on the 80 differentially expressed genes in rejection samples versus nonrejection samples (Supplemental Table 1) suggested a separation of the suspected AMR biopsy from TCMR biopsies along the first principal component (Figure 6A). These 80 genes were ranked using the absolute value of their loadings from the first principal component, which was thresholded at 0.1 . This identified 31 genes that contributed most to the variability between the AMR and TCMR episodes (Table 2 and Figure 6B). Genes that are upregulated in the biopsy collected during the suspected AMR episode include those associated with leukocyte-endothelial cell interaction (including ICAM1, VCAM1, and SELE), whereas genes that are upregulated in TCMRs include those associated with cytotoxicity (including $G Z M B$ ) (Figure 6, B and C). The greatest fold increase in AMR was for endothelial adhesion molecule ICAM1, and the greatest fold increase in TCMR was for GZMB (Table 2). This is consistent with the gene expression profiles of biopsies collected during TCMR episodes in 6 additional facial transplant recipients at our institution that suggest that TCMR is characterized by activation of cytotoxicity-associated genes (data not shown)

Validation of genes of interest using immunohistochemical staining of allograft biopsies. To validate the expression of the genes of interest at the protein level, immunohistochemical staining of ICAM1 (the greatest fold increase in AMR) and granzyme B (the greatest fold increase in TCMR) was carried out on facial
Figure 3. Banff histological grades of rejection for facial allograft biopsies and the immunosuppression regimen following transplantation. Triangles represent steroid pulses or the increase in the dose of the maintenance tacrolimus immunosuppression. Topical therapies are not shown. ATC, antithymocyte globulin; TPE, total plasma exchange; MMF, mycophenolate mofetil; AMR, antibody-mediated rejection; TCMR, T cell-mediated rejection.

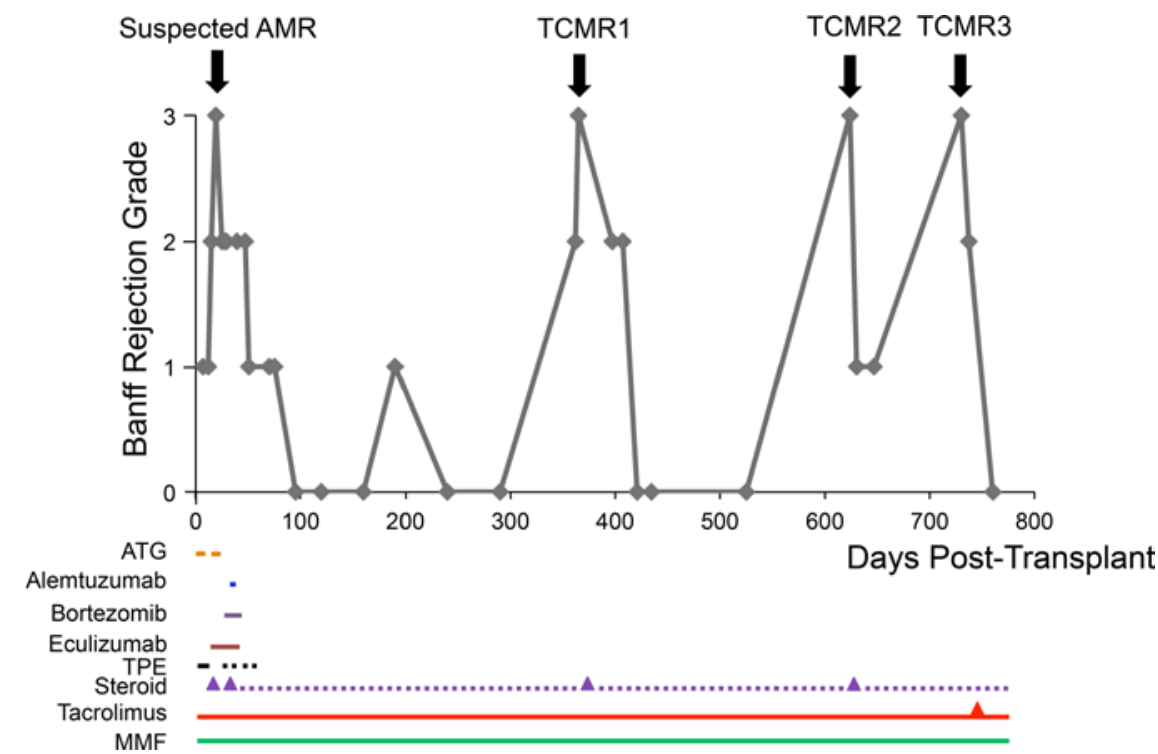




\section{B cells (Gated on CD19+ cells)}
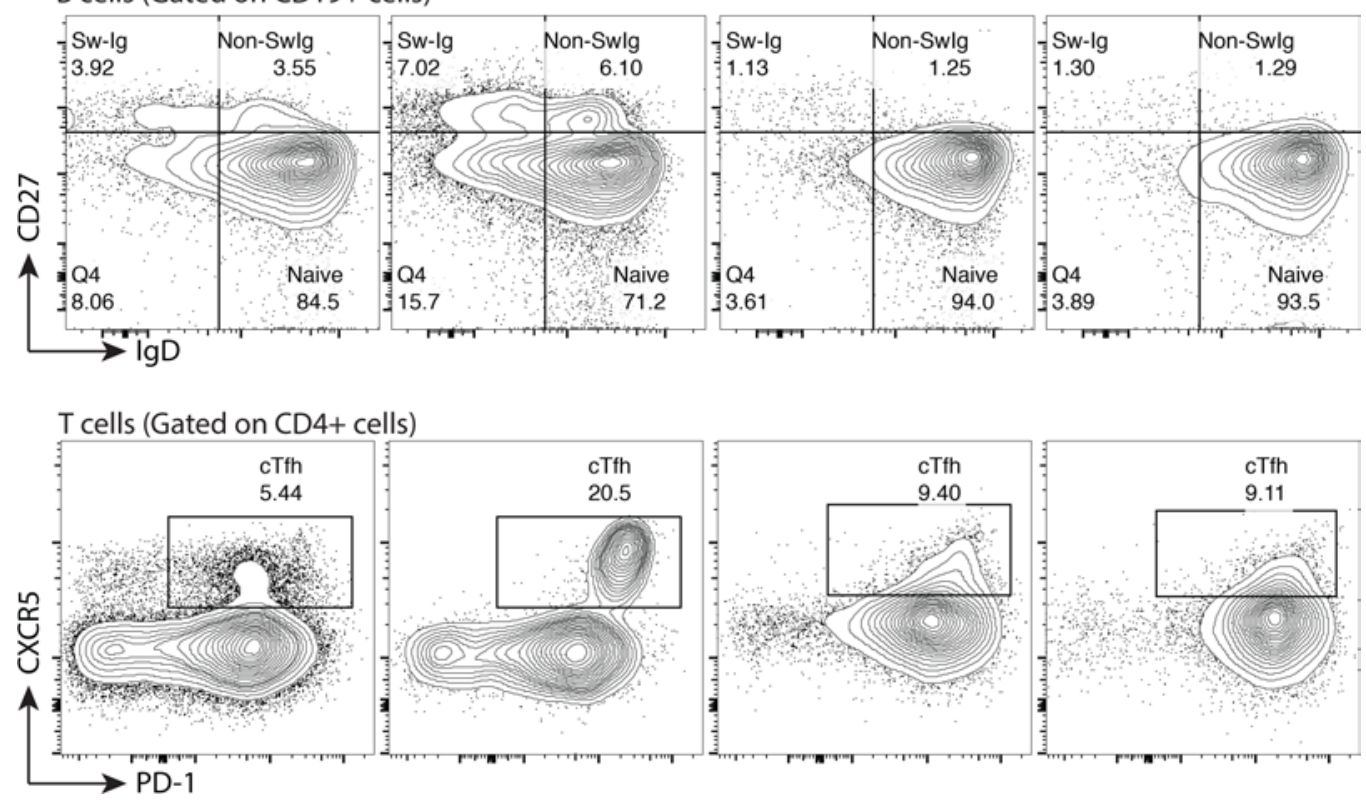

Figure 4. Dynamics of circulating B cells and $T$ follicular helper cells following facial transplantation. Contour plots of naive (CD19+CD27-lgD+), non-

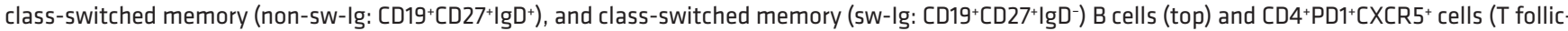
ular helper cells [cTfh]) (bottom) before transplant and at 1 week (suspected AMR), 22 months (nonrejection time point), and 24 months after transplant (TCMR3). Relative percentages of both non-sw-lg and sw-lg B cells and Tfh cells increased at 1 week after transplant, corresponding to the increase in DSAs and the suspected AMR episode.

allograft biopsies. AMR was associated with substantially more endothelial adhesion molecule ICAM1 expression in vessels compared with TCMR (Figure 6D). In contrast, granzyme B-positive cells were abundant in the allograft during TCMR but minimal during AMR.

\section{Discussion}

Here, we report the immunological characteristics of a highly sensitized recipient of a crossmatch-positive face transplant up to 4 years following transplantation. Immediately after transplantation, the patient had an episode of rejection, with an increase in circulating DSAs and strong C4d deposition within the allograft. After this rejection episode was controlled by an all-encompassing immunosuppressive regimen that included B cell-targeted therapies, the patient subsequently had 3 episodes of TCMR, which were successfully treated with either short steroid courses alone or an increase in maintenance immunosuppression. Currently, it has been 4 years since the patient underwent transplantation; the patient shows no signs of rejection and has a functioning allograft. Our case demonstrates two important findings. First, face transplantation in a highly sensitized patient with a positive preoperative donor-recipient crossmatch is possible and manageable. Second, our data provide proof of concept of the potential utility of molecular diagnostics to differentiate AMR from TCMR to guide clinical management and better understand the key molecular players in each rejection type.

Presensitization is a common scenario in VCA candidates because the clinical management of their

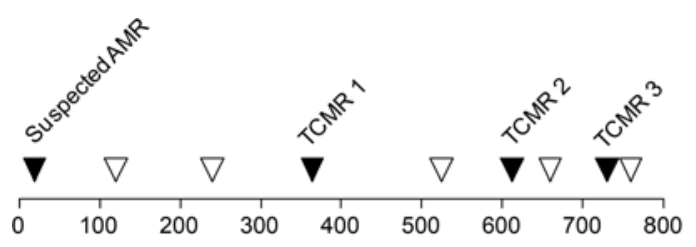

Days after transplantation
Rejection $\nabla$ Non-rejection
Figure 5. Timeline of facial allograft biopsies analyzed by the NanoString gene expression platform. Allograft biopsies collected during rejection (Banff grade 3 ) (black) $(n=4)$ or nonrejection (Banff grade 0) (white) ( $n=5)$ were analyzed by NanoString gene expression profiling platform. 

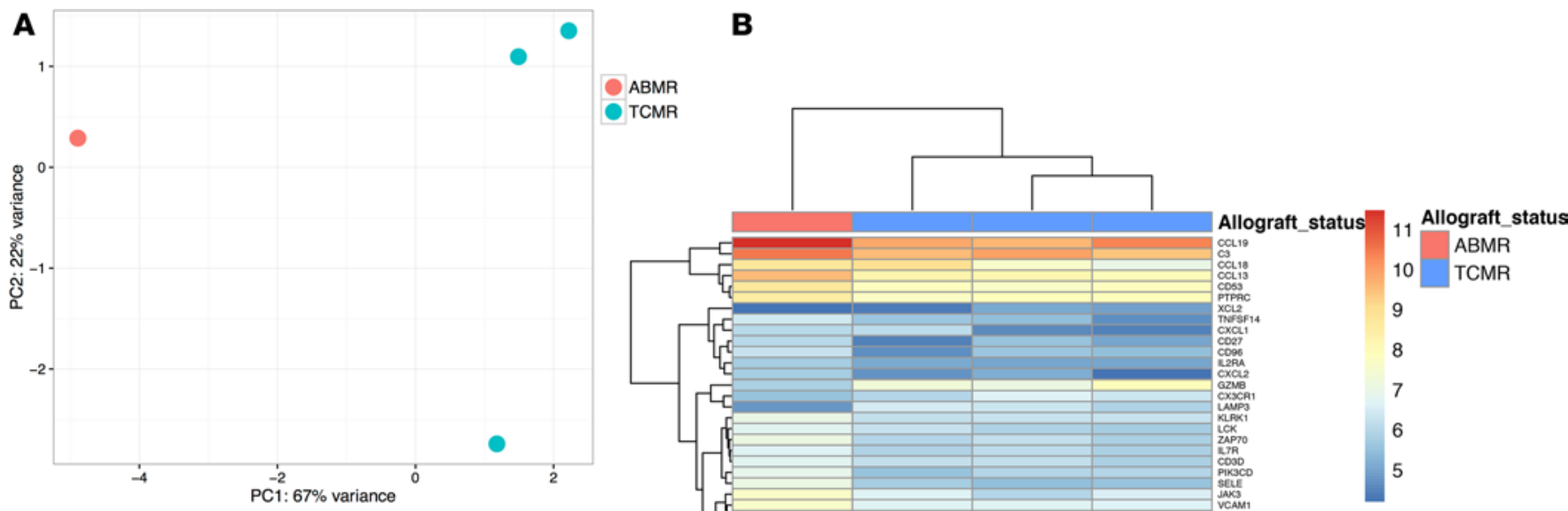

c

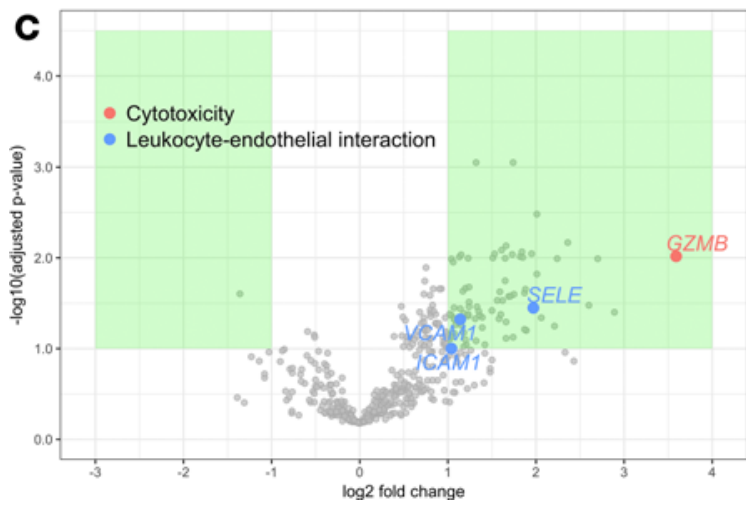

D

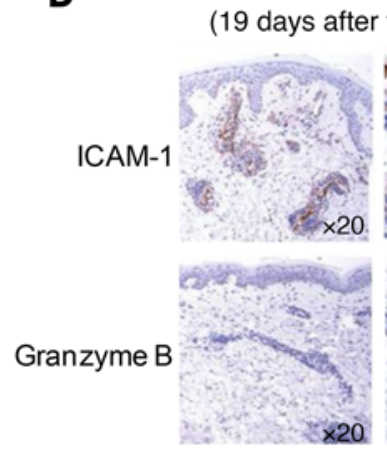

AMR

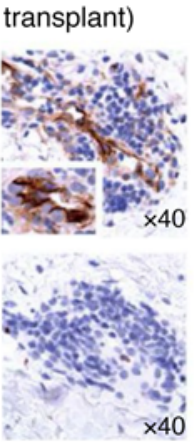

TCMR1

(12 months after transplant)

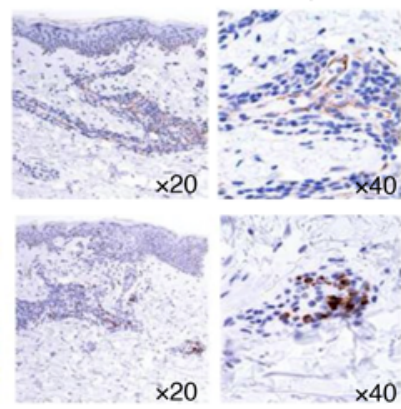

Figure 6. Gene expression profiling of allograft skin biopsies suggests distinctive features in the suspected AMR episode compared with TCMR episodes. (A) Unsupervised principal component analysis performed on 80 genes differentially expressed in a unpaired 2-tailed $t$ test comparing rejection ( $n=4$ ) to nonrejection $(n=5)$ samples (adjusted $P \leq 0.1)$ suggested a separation of the AMR biopsy from TCMR biopsies along the first principal component. (B) These 80 genes were ranked using the absolute value of their loadings from the first principal component, which was thresholded at 0.1 , to identify those contributing most to the observed variability between AMR and TCMR. This yielded 31 genes. The heatmap shows the expression of these 31 genes. Each column represents a facial allograft biopsy, labeled according to whether it was collected during the suspected AMR (red) or TCMR (blue) episodes. The expression of these genes clustered the samples according to the type of rejection. (C) In the volcano plot, the association strength ( $y$ axis) is compared with log fold change $\left(x\right.$ axis) in rejection $(n=4)$ versus nonrejection $(n=5)$ biopsies. The area shaded in green represents $\log _{2}$ fold change $\geq 1$ and adjusted $P \leq 0.1$. (D) Representative images of biopsies collected during the suspected AMR episode and the TCMR episode at 12 months following transplant. AMR was associated with substantially more endothelial adhesion molecule ICAM1 expression in vessels compared with TCMR. Bulging ICAM1-positive endothelial cells, morphology typical of endothelial activation, is shown in the inset. Granzyme B-positive cells were abundant in the allograft during TCMR but minimal during AMR. The staining was conducted in biopsies collected during the suspected AMR episode and each of the 4 episodes of TCMR. Original magnification, $\times 20$ (first and third columns); $\times 40$ (second and fourth columns); $\times 100$ (inset).

primary injuries, such as severe burns, frequently requires multiple blood transfusions and/or cadaveric skin grafts $(24,25)$. Before our case, there was little knowledge in the field of whether it is immunologically feasible to transplant a crossmatch-positive VCA allograft to a presensitized patient. Traditionally, a positive preoperative crossmatch is considered a contraindication to solid organ transplants $(26,27)$. Until our case, all reported VCAs have closely followed the crossmatch practices of solid organ transplantation and have been performed only in cases of negative preoperative crossmatches, resulting in prolonged waiting times for sensitized patients and even withdrawal or exclusion from the waiting list in some cases (28). In this report, our medium-term outcomes demonstrate that transplantation of a $\mathrm{T}$ and B cell crossmatch-positive facial allograft in the setting of a highly sensitized recipient is feasible. Although we are hopeful that our findings represent a step toward expanding the eligibility criteria for VCA transplantation for sensitized candidates, we acknowledge that evaluation of the long-term outcomes will be necessary to inform the eventual prognosis. 
Table 2. List of the top 31 ranked genes that showed the greatest contribution to the variability between AMR and TCMR

\begin{tabular}{|c|c|c|c|}
\hline Gene symbol & $\begin{array}{c}\log _{2} \text { fold change } \\
\text { (rejection vs. nonrejection) }\end{array}$ & $\begin{array}{l}\log _{2} \text { fold change } \\
\text { (AMR vs. TCMR) }\end{array}$ & $\begin{array}{l}\text { Loadings PC1 } \\
\text { (AMR vs. TCMR) }\end{array}$ \\
\hline ICAM1 & 1.04 & 1.62 & -0.246848081 \\
\hline GZMB & 3.59 & -1.57 & 0.244973925 \\
\hline SELE & 1.97 & 1.46 & -0.226106683 \\
\hline CCL19 & 2.21 & 1.52 & -0.222041372 \\
\hline CCL13 & 1.58 & 1.4 & -0.21852107 \\
\hline LAMP3 & 2.7 & -1.5 & 0.212371222 \\
\hline FCGR2A & 1.07 & 1.27 & -0.205564095 \\
\hline TNFSF14 & 1.5 & 1.15 & -0.19213774 \\
\hline$C Y B B$ & 1.18 & 1.27 & -0.19170439 \\
\hline JAK3 & 1.66 & 1.26 & -0.191632668 \\
\hline CXCL1 & 1.42 & 0.996 & -0.177472227 \\
\hline CXCL2 & 1.32 & 0.996 & -0.164396636 \\
\hline ZAP7O & 1.15 & 1.03 & -0.162032776 \\
\hline CCL18 & 2.89 & 0.839 & -0.158569039 \\
\hline PIKЗСD & 1.09 & 1.06 & -0.156521915 \\
\hline CD53 & 2.06 & 0.995 & -0.152613911 \\
\hline CD27 & 1.62 & 1 & -0.148181534 \\
\hline IRF1 & 1.23 & 1.01 & -0.145360441 \\
\hline CD96 & 1.18 & 0.982 & -0.140556932 \\
\hline FCER1G & 1.12 & 0.972 & -0.139750361 \\
\hline VCAM1 & 1.14 & 0.89 & -0.137939103 \\
\hline C3 & 1.07 & 0.846 & -0.134222817 \\
\hline LCK & 1.04 & 0.763 & -0.125562322 \\
\hline CX3CR1 & 1.24 & -0.83 & 0.125261455 \\
\hline IL7R & 2.01 & 0.767 & -0.121354198 \\
\hline$C D 3 D$ & 1.73 & 0.67 & -0.110537015 \\
\hline KLRK1 & 1.66 & 0.717 & -0.109647043 \\
\hline PTPRC & 1.85 & 0.699 & -0.106859494 \\
\hline CD40 & 1.23 & 0.649 & -0.103604743 \\
\hline IL2RA & 1.88 & 0.656 & -0.102615724 \\
\hline$X C L 2$ & 1.31 & -0.629 & 0.100246278 \\
\hline
\end{tabular}

Differentially expressed genes, comparing rejecting biopsies versus nonrejecting biopsies, were subjected to unsupervised principal component analysis (PCA) using normalized $\log _{2}$ mRNA expression values for the samples collected during the suspected AMR and TCMR episodes. The value of each gene loading in the PCA was used as a measure of its contribution to the sample clustering. The top 31 ranked genes using a cutoff of 0.1 for the absolute value of the loading were selected. PC, principal component.
Despite the presence of preformed antibodies and a positive preoperative $\mathrm{T}$ cell crossmatch, circulating DSAs were undetectable during the subsequent rejection episodes in our patient. This is in agreement with our hypothesis that cellular and molecular mechanisms underlying AMR and TCMR are different. Indeed, despite complete depletion of $\mathrm{T}$ effector cells after antithymocyte globulin in face transplant recipients early after transplant (23), AMR still occurred due to the presence of DSAs, likely triggering injury through complement activation and/or Fc-mediated cytotoxicity (8). Surprisingly, even though antithymocyte globulin targets multiple $\mathrm{T}$ cell markers, including CD3, circulating Tfh cells were preserved and an expansion of memory $\mathrm{B}$ cells was observed, potentially contributing to the antibody response, although the definitive role of circulating Tfh cells in DSA generation is unclear $(29,30)$. Interestingly, the lack of Tfh cell depletion was also present in other face transplant recipients treated with antithymocyte globulin at the first week after transplant (23). A possible explanation for the absence of DSAs during follow-up is related to the multiple (repeated) rejection treatments the patient received for AMR initially and then TCMRs. As DSA generation requires efficient $T$ cell help to $B$ cells, it is feasible that the humoral response was not provoked in the subsequent TCMRs due to lack of cognate help from the $\mathrm{T}$ cell population because of the prompt rejection treatments.

Given that different treatment strategies are required for AMR and TCMR, as demonstrated in our patient, there is a critical need to understand the features that can differentiate AMR from TCMR. We sought to investigate if gene expression profiling conducted directly in the grafted tissue can differentiate AMR from TCMR. First, we compared the gene expression profiles in rejection biopsies (regardless of

the type of rejection) with nonrejection biopsies. Of the 79 genes that were upregulated in rejection biopsies, we observed activation of the IFN- $\gamma$ signaling pathway, the CXCR3/CCR5 pathway, and the immune effector function genes, which have also been reported to be overexpressed in biopsies from solid organ transplants during rejection (31-34). This suggests that the perturbations in gene expression during rejection, induced by a complex cascade of cytokines and coordinate activation of specific pathways, may share similarities regardless of the allograft type.

Next, to identify the genes that contribute most to the differences between AMR and TCMR, we performed a principal component analysis using the differentially expressed genes identified from biopsies collected during the suspected AMR episode and the subsequent TCMRs. Our data suggested that there are distinctive features in AMR compared with TCMR within the facial allograft. Genes that were upregulated in the suspected AMR episode include ICAM1, VCAM1, and SELE, which are associated with endothelial activation and leukocyte-endothelial cell interactions $(35,36)$. This is consistent with previous studies of solid organ transplants, which showed that the main location of the antigens targeted in AMR is vascular endothelium, which 
when activated, upregulates vascular cell adhesion molecules, promoting leukocyte trafficking (8). Although it is tantalizing to hypothesize that a mechanism resembling antibody-dependent cell-mediated cytotoxicity is responsible for microcirculation injury and evokes the endothelial response to injury in AMR following VCA transplantation, we are cautious not to derive any generalized conclusions based on a single case.

Our study provides proof of concept of the potential utility of molecular diagnostics to aid in the diagnosis of AMR to guide clinical management. Furthermore, our case illustrates that gene expression profiling of allograft biopsies offers the potential for new insights into the mechanisms underlying VCA rejection. Finally, our medium-term outcomes demonstrate that face transplantation in a highly sensitized patient with a positive preoperative crossmatch is feasible and manageable. However, the high-cumulative dose of immunosuppression required to control the initial AMR event raises concern for potential longterm cancer and infectious complications. Despite our successful medium-term outcome in this case, we would advise caution in performing a transplant in a patient with a positive crossmatch at time of transplant. Strategies to reduce the rejection events after face transplantation are warranted, which may include tolerance protocols or the use of agents to promote immune regulation, such as low-dose IL-2 (37-39). A limitation of the present study is that our findings are derived from a single patient. The pitfalls of single-case analyses include concerns regarding the reliability and reproducibility (40), and we acknowledge that the extent to which our findings are generalizable is unclear. Additional research with an adequate number of patient samples to ensure statistical validity will be needed to test this notion. Given the paucity of VCA transplants and rarity of AMR, this will require a multicenter approach with consensus diagnostic criteria to enable robust conclusions.

\section{Methods}

RNA extracted from archival formalin-fixed, paraffin-embedded (FFPE) 4-mm skin punch biopsies was used to quantify the expression of 730 literature-derived genes using the NanoString platform (41) (NanoString Technologies). We conducted gene expression profiling on well-annotated archival FFPE biopsies because it allowed molecular testing to be carried out using the same biopsy sample used for corresponding histological examination, allowing for direct histomolecular comparison.

The NanoString platform is hybridization-based and does not require reverse transcription of mRNA and subsequent cDNA amplification (41). This feature of NanoString technology offers an advantage over PCR-based methods in yielding superior gene expression quantification (42). For histological analyses, paraffin sections from the same FFPE blocks were examined according to the 2007 Banff classification (7) by a pathologist without the knowledge of the molecular findings. Immunohistochemistry for ICAM1 and granzyme B was performed using paraffin sections from the same FFPE blocks. Flow cytometric analysis was carried out using peripheral blood mononuclear cells isolated from blood samples collected prior to transplant and at serial time points following transplantation. Serum samples were tested for the presence of circulating donor-specific anti-HLA antibodies using the single antigen bead-based assay (One Lambda) on a Luminex platform. See Supplemental Methods for details.

Statistics. The mean values were calculated from the normalized $\log _{2}$ mRNA expression values from biopsies during rejection and nonrejection. Differentially expressed genes (Supplemental Table 1) were evaluated by unpaired 2-tailed $t$ test, using the $R$ statistical package ( $R$ version 3.2.3). Adjusted $P$ values were calculated using false discovery rate. Genes with fewer than 20 counts in $50 \%$ or more of the samples were filtered to remove low-abundance transcripts. Genes with adjusted $P$ values of $\leq 0.1$ were considered as differentially expressed and were considered to be significant.

Study approval. The patient provided written informed consent to participate in the clinical trial (ClinicalTrials.gov number, NCT01281267) for face transplantation, as approved by the Partners Human Research Committee at Brigham and Women's Hospital (2008P000550). In addition, the patient provided written informed consent for use of photographs in the manuscript.

\section{Author contributions}

TSW and NM cowrote the manuscript, performed the experiments, and acquired and analyzed the data. AC, GM, CL, SGT, and BP contributed patient samples, analyzed the data, and contributed to the writing of the manuscript. VB, SHS, and DS performed the statistical and bioinformatics analysis and contributed to the writing of the manuscript. JT, EB, RAC, and TJB supervised the data analysis and critically revised the manuscript. LVR conceived of the study and wrote the final version of the manuscript. 


\section{Acknowledgments}

This paper is dedicated to the patient, whose commitment to advancing VCA research made this study possible. This work was supported by the Assistant Secretary of Defense and Health Affairs, through Reconstructive Transplant Research, under award W81XWH-16-1-0647 (to LVR), W81XWH-16-1-0689 (to LVR), W81XWH-13-2-0053 (to BP), and W911QY-09-C-0216 (to BP). Opinions, interpretations, conclusions, and recommendations are those of the author and are not necessarily endorsed by the Department of Defense. TSW is supported by the American Society of Transplantation's Transplantation and Immunology Research Network Fellowship Research Grant. NM is supported by NIH grant T32 DK007527. Biostatistics and bioinformatics support was funded by the Harvard Catalyst I The Harvard Clinical and Translational Science Center (through NIH grant UL1 RR 025758). The content is solely the responsibility of the authors and does not necessarily represent the official views of the NIH.

Address correspondence to: Leonardo V. Riella, Brigham and Women's Hospital, 75 Francis Street, Boston, Massachusetts 02115, USA. Phone: 617.732.5500; Email: 1riella@bwh.harvard.edu.

1. Langer R, Vacanti JP. Tissue engineering. Science. 1993;260(5110):920-926.

2. Pomahac B, et al. Three patients with full facial transplantation. N Engl J Med. 2012;366(8):715-722.

3. Pomahac B, et al. Restoration of facial form and function after severe disfigurement from burn injury by a composite facial allograft. Am J Transplant. 2011;11(2):386-393.

4. Sosin M, Rodriguez ED. The face transplantation update: 2016. Plast Reconstr Surg. 2016;137(6):1841-1850.

5. Weissenbacher A, Loupy A, Chandraker A, Schneeberger S. Donor-specific antibodies and antibody-mediated rejection in vascularized composite allotransplantation. Curr Opin Organ Transplant. 2016;21(5):510-515.

6. Petruzzo P, Dubernard JM. The International Registry on Hand and Composite Tissue allotransplantation. Clin Transpl. 2011:247-253.

7. Cendales LC, et al. The Banff 2007 working classification of skin-containing composite tissue allograft pathology. Am J Transplant. 2008;8(7):1396-1400.

8. Colvin RB, Smith RN. Antibody-mediated organ-allograft rejection. Nat Rev Immunol. 2005;5(10):807-817.

9. Seemayer CA, Gaspert A, Nickeleit V, Mihatsch MJ. C4d staining of renal allograft biopsies: a comparative analysis of different staining techniques. Nephrol Dial Transplant. 2007;22(2):568-576.

10. Loupy A, et al. Significance of C4d Banff scores in early protocol biopsies of kidney transplant recipients with preformed donor-specific antibodies (DSA). Am J Transplant. 2011;11(1):56-65.

11. Szymańska S, Grajkowska W, Sobieszczańska-Małek M, Zieliński T, Pronicki M. Reclassification of C4d-Positive Endomyocardial Biopsy (EMB) According to New International Society for Heart and Lung Transplantation (ISHLT) 2013 Categories for Reporting Pathologic Antibody-Mediated Rejection (pAMR): Preliminary Data from a Polish Single-Center Study. Ann Transplant. 2015;20:351-356.

12. Halloran PF, Merino Lopez M, Barreto Pereira A. Identifying subphenotypes of antibody-mediated rejection in kidney transplants. Am J Transplant. 2016;16(3):908-920.

13. Berry GJ, et al. The 2013 International Society for Heart and Lung Transplantation Working Formulation for the standardization of nomenclature in the pathologic diagnosis of antibody-mediated rejection in heart transplantation. J Heart Lung Transplant. 2013;32(12):1147-1162.

14. Haas M, et al. Banff 2013 meeting report: inclusion of c4d-negative antibody-mediated rejection and antibody-associated arterial lesions. Am J Transplant. 2014;14(2):272-283.

15. Weissenbacher A, et al. Antibody-mediated rejection in hand transplantation. Transpl Int. 2014;27(2):e13-e17.

16. Schneeberger S, et al. Upper-extremity transplantation using a cell-based protocol to minimize immunosuppression. Ann Surg. 2013;257(2):345-351.

17. Kanitakis J, et al. Absence of c4d deposition in human composite tissue (hands and face) allograft biopsies: an immunoperoxidase study. Transplantation. 2007;84(2):265-267.

18. Landin L, Cavadas PC, Ibañez J, Roger I, Vera-Sempere F. CD3+-mediated rejection and C4d deposition in two composite tissue (bilateral hand) allograft recipients after induction with alemtuzumab. Transplantation. 2009;87(5):776-781.

19. Kaufman CL, et al. Graft vasculopathy in clinical hand transplantation. Am J Transplant. 2012;12(4):1004-1016.

20. Petruzzo P, et al. Long-term follow-up in composite tissue allotransplantation: in-depth study of five (hand and face) recipients. Am J Transplant. 2011;11(4):808-816.

21. Magro CM, Dyrsen ME. The use of C3d and C4d immunohistochemistry on formalin-fixed tissue as a diagnostic adjunct in the assessment of inflammatory skin disease. J Am Acad Dermatol. 2008;59(5):822-833.

22. Chandraker A, et al. The management of antibody-mediated rejection in the first presensitized recipient of a full-face allotransplant. Am J Transplant. 2014;14(6):1446-1452.

23. Borges TJ, et al. Codominant role of interferon- $\gamma$ - and interleukin-17-producing t cells during rejection in full facial transplant recipients. Am J Transplant. 2016;16(7):2158-2171.

24. Win TS, Frew Q, Taylor CJ, Peacock S, Pettigrew G, Dziewulski P. Allosensitization following skin allografts in acute burn management: Are burns patients suitable face transplant candidates? J Plast Reconstr Aesthet Surg. 2015;68(8):1155-1157.

25. Duhamel P, et al. Anti-HLA sensitization in extensively burned patients: extent, associated factors, and reduction in potential access to vascularized composite allotransplantation. Transpl Int. 2015;28(5):582-593. 
26. Patel R, Terasaki PI. Significance of the positive crossmatch test in kidney transplantation. N Engl J Med. 1969;280(14):735-739.

27. Tait $\mathrm{BD}$, et al. Consensus guidelines on the testing and clinical management issues associated with HLA and non-HLA antibodies in transplantation. Transplantation. 2013;95(1):19-47.

28. Lantieri L, et al. Feasibility, reproducibility, risks and benefits of face transplantation: a prospective study of outcomes. $A m J$ Transplant. 2011;11(2):367-378.

29. Ueno H, Banchereau J, Vinuesa CG. Pathophysiology of T follicular helper cells in humans and mice. Nat Immunol. 2015;16(2):142-152.

30. Walters GD, Vinuesa CG. T follicular helper cells in transplantation. Transplantation. 2016;100(8):1650-1655.

31. Hidalgo LG, Halloran PF. Role of IFN-gamma in allograft rejection. Crit Rev Immunol. 2002;22(4):317-349.

32. Shin $\mathrm{H}$, et al. Longitudinal analysis of whole blood transcriptomes to explore molecular signatures associated with acute renal allograft rejection. Bioinform Biol Insights. 2014;8:17-33

33. Halloran PF, Famulski KS, Reeve J. Molecular assessment of disease states in kidney transplant biopsy samples. Nat Rev Nephrol. 2016;12(9):534-548.

34. Spivey TL, et al. Gene expression profiling in acute allograft rejection: challenging the immunologic constant of rejection hypothesis. J Transl Med. 2011;9:174.

35. Valujskikh A, Heeger PS. Emerging roles of endothelial cells in transplant rejection. Curr Opin Immunol. 2003;15(5):493-498

36. Drachenberg CB, Papadimitriou JC. Endothelial injury in renal antibody-mediated allograft rejection: a schematic view based on pathogenesis. Transplantation. 2013;95(9):1073-1083.

37. Leonard DA, et al. Vascularized composite allograft tolerance across MHC barriers in a large animal model. Am J Transplant. 2014;14(2):343-355.

38. Lin CH, et al. Combined Anti-CD154/CTLA4Ig costimulation blockade-based therapy induces donor-specific tolerance to vascularized osteomyocutaneous allografts. Am J Transplant. 2016;16(7):2030-2041.

39. Murakami N, et al. The effect of low-dose IL-2 in clinical facial transplantation: Molecular and immunological response [abstract B293]. Am J Transplant. 2017;17:(suppl 3).

40. Brannon AR, Sawyers CL. "N of 1" case reports in the era of whole-genome sequencing. J Clin Invest. 2013;123(11):4568-4570

41. Geiss GK, et al. Direct multiplexed measurement of gene expression with color-coded probe pairs. Nat Biotechnol. 2008;26(3):317-325.

42. Reis PP, et al. mRNA transcript quantification in archival samples using multiplexed, color-coded probes. BMC Biotechnol. 2011;11:46 